\title{
Weak intermolecular interactions in an ionically bound molecular adsorbate: cyclopentadienyl=Cu(111)
}

Article

Published Version

Hedgeland, H., Lechner, B. A. J., Tuddenham, F. E., Jardine, A. P., Allison, W., Ellis, J., Sacchi, M., Jenkins, S. J. and Hinch, B. J. (2011) Weak intermolecular interactions in an ionically bound molecular adsorbate:

cyclopentadienyl=Cu(111). Physical Review Letters, 106 (18). 186101. ISSN 0031-9007 doi:

https://doi.org/10.1103/PhysRevLett.106.186101 Available at https://centaur.reading.ac.uk/39059/

It is advisable to refer to the publisher's version if you intend to cite from the work. See Guidance on citing.

To link to this article DOI: http://dx.doi.org/10.1103/PhysRevLett.106.186101

Publisher: American Physical Society

All outputs in CentAUR are protected by Intellectual Property Rights law, including copyright law. Copyright and IPR is retained by the creators or other copyright holders. Terms and conditions for use of this material are defined in the End User Agreement. 


\section{CentAUR}

Central Archive at the University of Reading

Reading's research outputs online 


\title{
Weak Intermolecular Interactions in an Ionically Bound Molecular Adsorbate: Cyclopentadienyl $/ \mathrm{Cu}(\mathbf{1 1 1})$
}

\author{
H. Hedgeland, ${ }^{1, *}$ B. A. J. Lechner, ${ }^{1}$ F. E. Tuddenham, ${ }^{1}$ A. P. Jardine, ${ }^{1}$ W. Allison, ${ }^{1}$ J. Ellis, ${ }^{1}$ M. Sacchi, ${ }^{2}$ \\ S. J. Jenkins, ${ }^{2}$ and B. J. Hinch ${ }^{3}$ \\ ${ }^{1}$ Cavendish Laboratory, University of Cambridge, JJ Thomson Avenue, Cambridge, CB3 OHE, United Kingdom \\ ${ }^{2}$ Department of Chemistry, University of Cambridge, Lensfield Road, Cambridge, CB2 1EW, United Kingdom \\ ${ }^{3}$ Department Chemistry and Chemical Biology, Rutgers University, Piscataway, New Jersey 08854, USA
}

(Received 28 January 2011; published 2 May 2011)

\begin{abstract}
The dissociative adsorption of cyclopentadiene $\left(\mathrm{C}_{5} \mathrm{H}_{6}\right)$ on $\mathrm{Cu}(111)$ yields a cyclopentadienyl $(\mathrm{Cp})$ species with strongly anionic characteristics. The $\mathrm{Cp}$ potential energy surface and frictional coupling to the substrate are determined from measurements of dynamics of the molecule together with density functional calculations. The molecule is shown to occupy degenerate threefold adsorption sites and molecular motion is characterized by a low diffusional energy barrier of $40 \pm 3 \mathrm{meV}$ with strong frictional dissipation. Repulsive dipole-dipole interactions are not detected despite charge transfer from substrate to adsorbate.
\end{abstract}

DOI: 10.1103/PhysRevLett.106.186101

The net charge and dipole moment of an organic adsorbate are determining factors in the alignment between electronic states of the adsorbed molecule and the substrate $[1,2]$. Electrostatic rearrangements at the interface also control long-range forces and hence self-organization during the early stages of thin-film growth [3,4]. Strongly bound ionic species, together with appropriate functionalization, offer a range of new possibilities for self-assembly but little is known of their behavior. We provide a quantitative understanding of anionically adsorbed systems through a study of dynamical behavior. The methods deliver information, for example, on intermolecular forces, that is not readily available from conventional spectroscopic methods. Our results reveal not only the adsorption site and the extent of electronic rearrangement but also the dissipative coupling responsible for the mechanism of molecular transport.

Cationic adsorbates, such as the alkali metals, are dominated by charge donation and a strong surface dipole. The behavior of species that accept electrons from the substrate is less well understood [3-5]. It is oversimplistic to associate work-function changes with the adsorbate charge, and consequently atomic adsorbates $[5,6]$ can behave differently from molecular adsorbates [3]. Intermolecular forces between cations are well understood but there are no dynamical measurements at present for extended molecular systems that adsorb anionically.

We have studied what should be a classic anionic system. Cp is a well-known ligand in organometallic chemistry, useful in controlling the oxidation state of isolated metal ions. When adsorbed on a surface, $\mathrm{Cp}$ is a prototypical organic ionic species. We determine $\mathrm{Cp}$-substrate and $\mathrm{Cp}-\mathrm{Cp}$ interactions via the effect they have on the thermally induced motion of $\mathrm{Cp}$ using helium spin echo (HeSE). The thermal activation of the diffusion and the directional dependence of the HeSE signal indicate the
PACS numbers: 68.43. $-\mathrm{h}$, 68.35.Fx, 68.35.Ja, 68.49.Bc

adsorbate-substrate potential, and the correlated motion of adsorbates controlled by the lateral interactions. The method is uniquely sensitive to such interactions, for example, in cationic adsorption where they are present [7] and also when they are weak or absent [8].

$\mathrm{Cp}$ is formed by exposure of the $\mathrm{Cu}(111)$ surface to monomeric $\mathrm{C}_{5} \mathrm{H}_{6}$ at temperatures above $150 \mathrm{~K}$. One hydrogen atom is lost on adsorption, resulting in an adsorbed species showing a high degree of anionicity, which remains stable to $>400 \mathrm{~K}$, while $\mathrm{H}_{2}$ desorption occurs around room temperature [9]. A single crystal $\mathrm{Cu}(111)$ sample, mounted in a chamber of base pressure $5 \times 10^{-11} \mathrm{mbar}$, was prepared by cycles of $\mathrm{Ar}^{+}$sputtering $(800 \mathrm{eV})$ and annealing at $800 \mathrm{~K}$, with surface quality monitored using the helium reflectivity. $\mathrm{Cp}$ was deposited on the surface by backfilling the chamber with $\mathrm{C}_{5} \mathrm{H}_{6}$, previously cleaned by freeze-thaw cycles, and monitoring the drop in the specularly reflected helium beam during adsorption at $300 \mathrm{~K}$. All experiments were carried out with reflectivity drops of $65 \%$. Assuming an effective cross section for the Cp of $200 \AA^{2}$ [10] results in an absolute coverage estimate of 0.03 monolayers, achievable with a reproducibility of better than $5 \%$. The HeSE technique is now well established and a detailed description of the technique has been given elsewhere, in particular, [11,12]. Essentially, helium atoms scatter from the mobile $\mathrm{Cp}$ species on the surface and the technique provides a measurement of correlations in the $\mathrm{Cp}$ positions with time. An individual measurement gives the time decay of the correlation function, $I(\Delta \mathbf{K}, t)$, for particular momentum transfers, $\Delta \mathbf{K}$, of the scattered particles. $I(\Delta \mathbf{K}, t)$ is the well-known intermediate scattering function (ISF) $[13,14]$ and represents the rate of "dephasing" of the Cp positions over the periodic repeat length $2 \pi / \Delta \mathbf{K}$ with times, $t$, in the picosecond range. By examining the variation of the ISF across a range of experimental conditions (varying temperature, scattering direction, and 
$\Delta \mathbf{K}$ ), we build up a detailed, microscopic picture of motion on the surface [12].

Typical HeSE measurements of $\mathrm{Cp}$ at room temperature are shown in Fig. 1 as points, for the two principal lattice directions. The high mobility of $\mathrm{Cp}$ is immediately apparent from the decay of $I(\Delta \mathbf{K}, t)$ over a few picoseconds. In general, we see that the decays are made up of several distinct components. The majority of the measurement is dominated by a loss of correlation due to $\mathrm{Cp}$ diffusion, seen in Fig. 1, with a characteristic time scale of order 2 ps. Along the $[1 \overline{1} 0]$ direction a single exponential decay (solid blue line) describes the data, as in most simple models of diffusion [12]. However, along the [112̄] azimuth, at parallel momentum transfers above $0.6 \AA^{-1}$, two distinct exponentially decaying components are seen [Fig. 1(b)].

Nonexponential forms of the ISF could arise from ballistic motion or rotation. However, the presence of multiple decays over a particular range of $\Delta \mathbf{K}$ is not consistent with ballistic processes [15] and the coupling of $\mathrm{He}$ atoms to rotational motion of a $\mathrm{Cp}$ anion is not anticipated, as the giant scattering cross section of the anion will have nearcylindrical symmetry at the low helium energies involved $(8 \mathrm{meV})$ [10]. The observation of distinct exponential terms on similar time scales suggests strongly hopping between sites forming a non-Bravais lattice $[16,17]$. The observation of a two-component line shape along [112̄] , but not along [110], strongly suggests that Cp hops between both the fcc and hep hollow sites. Although predicted, such line shapes

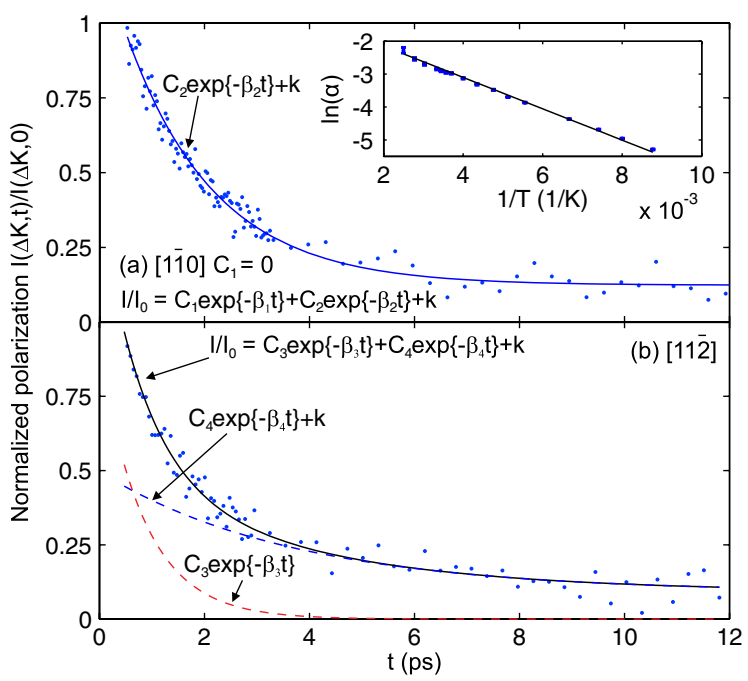

FIG. 1 (color online). Experimental ISFs measured along the (a) $[1 \overline{1} 0]$ and (b) $[11 \overline{2}]$ directions at $\Delta \mathbf{K} \sim 1.9 \AA^{-1}$ at room temperature (points). Along the [1ํㅣㄹ azimuth, a single exponential decay is seen (solid blue line), in agreement with the expected form for jump diffusion. However, along [112] the line shape is the sum of two exponential decays, indicating jumps between sites forming a non-Bravais lattice (the solid black line indicates the sum of dashed red and blue components). Data below $0.5 \mathrm{ps}$ are heavily influenced by phonons and are excluded. The inset shows the temperature dependency of the dephasing rate, at $\Delta \mathbf{K}=0.4 \AA^{-1}$ along [11̄̄], as an Arrhenius plot, giving an activation energy of $41 \pm 1 \mathrm{meV}$ for diffusion. have not previously been observed in surface experiments. The relative amplitude of the two components is known [16], which allows the dephasing rates, $\alpha$, to be obtained from the data independently. Values are shown in Fig. 2 (points) and show excellent agreement with the $\Delta \mathbf{K}$ dependence of the analytical model from [16], establishing the diffusion mechanism as nearest neighbor hopping between degenerate fcc and hcp hollow sites (solid lines), with a jump rate of $0.6 \mathrm{ps}^{-1}$ at $300 \mathrm{~K}$ [from Eq. (20), Ref. [16] ]. As with most models of diffusion, we expect an activated temperature dependence. The inset to Fig. 1 shows an Arrhenius plot for surface temperatures between 114 and $400 \mathrm{~K}$, yielding an effective activation energy of $41 \pm 1 \mathrm{meV}$. Pairwise intermolecular forces arising from electrostatic, van der Waals, or other interactions would be apparent in the data of Fig. 2 through dips at particular values of $\Delta \mathbf{K}$, as observed with alkali metals $[7,18]$. The absence of such features indicates these interactions are unimportant in the regime of measurement.

In order to further examine the $\mathrm{Cp}$ adsorption geometry, dynamics, and charge distributions, we performed first principles simulations. Various binding sites, adsorption geometries, and orientations have been investigated within a density functional theory generalized gradient approximation (DFT-GGA) approach, using the CASTEP code [19] and Perdew Wang (PW91) exchange-correlation functional [20]. A plane wave basis set expanded to an energy cutoff of $300 \mathrm{eV}$ was used to describe the electronic wave functions with integration over the Brillouin zone by summation over a $4 \times 4 \times 1$ Monkhorst-Pack mesh. A seven layer $\mathrm{Cu}$ slab was used with 19 vacuum layers; the top 4 layers and adsorbed molecules were allowed to relax fully [21]. For all high symmetry binding sites, the carbon ring remains planar and oriented parallel to the surface. The

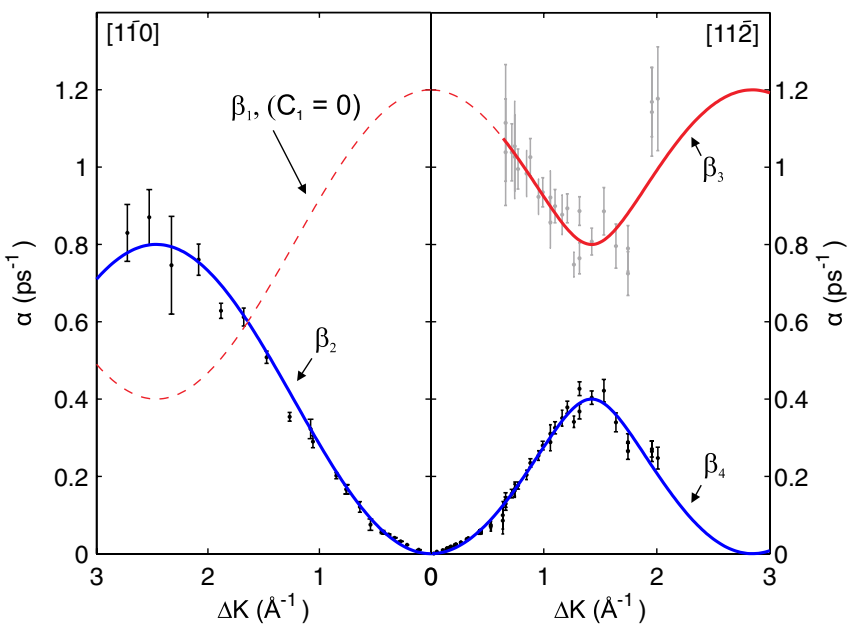

FIG. 2 (color online). Momentum transfer dependencies of ISF

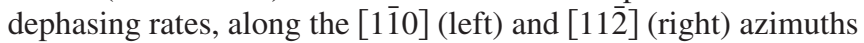
at $300 \mathrm{~K}$, shown as points. Solid lines indicate dephasing rates from an analytical model of jumps between degenerate fcc and hcp hollow sites (the dashed line shows where the second exponential term has zero intensity in the model). 
binding energies show some sensitivity to the azimuthal orientation and indicate energy barriers to rotational reorientation on fcc sites are less than $53 \mathrm{meV}$, while hop sites show barriers of $\sim 0.01 \mathrm{eV}$. The calculated binding energies, with respect to the gas phase $\mathrm{Cp}$ radical, at four high symmetry sites (hcp, fcc, bridge, atop) are 1.732, $1.732,1.671$, and $1.198 \mathrm{eV}$, respectively, for a relaxed $p(2 \sqrt{3} \times 2 \sqrt{3})$ phase. These support the experimental result that the ground state energies for the fcc and hop sites are most stable and degenerate, while the transition state in diffusion is the bridge site, with a barrier of $55 \mathrm{meV}$.

The DFT results confirm that close to 1 electron is transferred to the $\mathrm{Cp}$ radical, so the resultant charge magnitude and distribution closely resemble those of the aromatic gas phase anion. The $\mathrm{Cp}$ is largely ionically bound to the metallic substrate and we found that all the molecular orbitals of the adsorbed species, up to the highest occupied molecular orbital, have essentially the same energies as those of the isolated gas phase anion. There is evidence of spreading of the highest occupied and lowest unoccupied molecular orbitals, meaning that these are effectively the only orbitals that interact with the surface $d$ states, and indicate only a weak degree of covalency in the surface bonding, confirmed by overlap population analysis. Figure 3 (inset) illustrates the calculated electron accumulation and depletion around the adsorbed $\mathrm{Cp}$ molecule while Table I gives a quantitative summary of the adsorption properties obtained using DFT. While there is a net negative charge on the molecule, the dipole moment of the $\mathrm{Cp}$ is smaller in magnitude than a comparable atomic cationic species [18] and long-range dipolar mutual interactions will also be small. We note the adsorbate counterintuitively reduces the work function; the reduction is dominated by the cushion effect, demonstrated previously by benzene and cyclohexane [2]. Calculations of Cp species at higher densities (Table I) give only small variations in binding energies $(0.1 \mathrm{eV}), \mathrm{C}$-Cu distance $(0.05 \AA)$, and $\mathrm{H}$ tilt angles $\left(0.4^{\circ}\right)$, although the dipole moment changes by as much as $33 \%$. We conclude the $\mathrm{Cp}$ anion is ionically chemisorbed, with intermolecular interactions of electrostatic origin weaker than for a pointlike species.

To facilitate direct comparison between the experimental data and the energy barriers obtained using the DFT calculations, we performed a series of combined Langevin

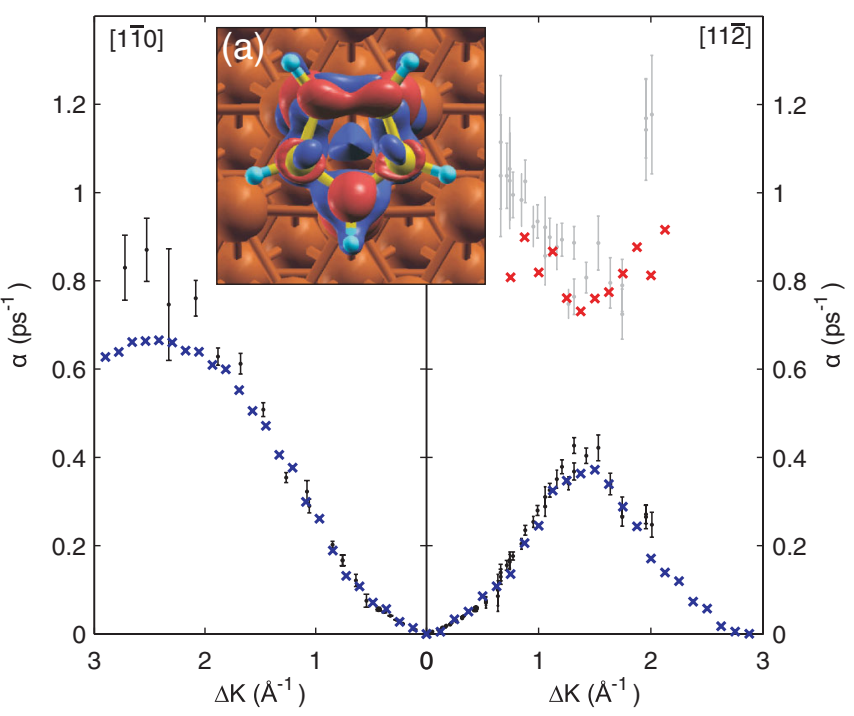

FIG. 3 (color). The main figure shows experimental data from Fig. 2 as points while crosses illustrate the best fit rates extracted from Langevin molecular-dynamics simulation data using an optimized PES and adsorbate-substrate friction (see text), with intermolecular interaction strengths as deduced from DFT calculations. Inset (a) shows surface adsorbed $\mathrm{Cp}$ with electron accumulation (red) and depletion (blue) relative to the gas phase neutral species as deduced from DFT calculations.

molecular dynamics and scattering simulations; we aim to reproduce the experimental data using the simplest model that includes all the relevant physical processes. The simulations trace the $\mathrm{Cp}$ motion over a trial twodimensional adiabatic potential energy surface (PES), using the functional form of the PES that has been successfully used several times before [8]. Adsorbate-substrate energy transfer and damping is modeled through a single friction parameter according to fluctuation-dissipation theory [12,22]. Interactions between $\mathrm{Cp}$ species are included using the coverage-dependent dipole moment arising from our DFT calculations within the Lau and Kohn dipole repulsion potential [23] and using a Topping model [24]. A PES with minima at the hollow sites, a hollow-tobridge barrier of $40 \pm 3 \mathrm{meV}$, and a friction parameter $\eta=2.5 \pm 0.5 \mathrm{ps}^{-1}$ gives best agreement with experiment, including the temperature dependence, and the simulated results are shown in Fig. 3.

TABLE I. Properties of adsorbed $\mathrm{Cp}$ deduced from DFT calculations at varying surface coverages.

\begin{tabular}{lccc}
\hline \hline & $(\sqrt{7} \times \sqrt{7}) R 19.1^{\circ}$ & $(3 \times 3)$ & $(2 \sqrt{3} \times 2 \sqrt{3}) R 30^{\circ}$ \\
\hline Total charge C (e) & -1.93 & -1.94 & -1.96 \\
Total charge H (e) & 0.76 & 0.78 & 0.83 \\
Work-function change $(\mathrm{eV})$ & -1.03 & -0.98 & -0.80 \\
Dipole moment (Debye) & -1.09 & -1.33 & -1.45 \\
Cp adsorption height $(\AA)$ & 2.30 & 2.26 & 2.25 \\
H-C angle $\left({ }^{\circ}\right)$ & 10.3 & 10.2 & 10.6 \\
Adsorption energy $(\mathrm{eV})$ & 1.61 & 1.66 & 1.73 \\
\hline \hline
\end{tabular}


In this case, the limiting energy barrier to diffusion and the effective "Arrhenius" activation are the same, although in general this is not so [12]. The energy barrier of the PES deduced from experiment agrees with the DFT calculation to within $15 \mathrm{meV}$. It would seem high mobility arises as the lateral extent of the $\mathrm{C}$ ring exceeds the natural periodicity of the substrate cores, and because the molecular orbitals of the fivefold symmetric adsorbate cannot overlap efficiently with the Bloch functions of the metal centers. The magnitude of the friction is higher than seen previously for alkali metals on a $\mathrm{Cu}(100)$ surface ( $\mathrm{Na}$ [7], $\eta=0.50 \mathrm{ps}^{-1} ; \mathrm{K}$ [18], $\eta=0.22 \mathrm{ps}^{-1}$; Cs [12], $\eta=$ $0.03 \mathrm{ps}^{-1}$ ) and is more similar to that seen for benzene adsorbed on a graphite substrate [25] $\left(\eta=2.2 \mathrm{ps}^{-1}\right)$, implying that the extended nature of the molecule encourages a significant phononic contribution to the adsorbatesubstrate energy transfer. It is interesting to note that the rate of lateral diffusion in this system corresponds almost exactly to the transition state theory limit [26], as the friction value maximizes the absolute jump rates for this PES. There is no evidence in Fig. 3 for strong intermolecular forces. We conclude the cushion effect nearly balances opposing dipole contribution terms, thereby preventing large repulsive interactions between $\mathrm{Cp}$ at this coverage and correlated motion of $\mathrm{Cp}$.

In summary, we find $\mathrm{Cp}$, a prototypical ionically bound molecular adsorbate, is remarkably mobile on $\mathrm{Cu}(111)$, yet remains strongly bound to the surface with excellent thermal stability due to a high level of ionic bonding; the ratio of binding energy to diffusion barrier is around 30. The high mobility can be attributed to the incompatible symmetry of $\mathrm{Cp}$ and $\mathrm{Cu}(111)$ combined with a high adsorbatesubstrate frictional interaction. We established a single jump mechanism between degenerate fcc and hcp hollow sites, the first observation of jumping on any non-Bravais surface lattice. At the coverages studied, long-range repulsive correlations in the motion of $\mathrm{Cp}$ are unexpectedly absent. Such long-range dipole-dipole interactions are minimized by the strong polarizability of the metal face and the cushion effect, which simultaneously results in a counterintuitive direction to the surface dipole moment. This archetypical ionic molecular adsorbate therefore shows a combination of physical properties that offer potential benefits. Reduced repulsion between neighboring molecules should enable the formation of unusually high density films. In principle, new classes of organic films could be possible, using functionalized species, where strong ionic binding provides stability and ability to withstand repeated high temperature cycling.

*hh242@cam.ac.uk

[1] G. Szulczewski, S. Sanvito, and G. Coey, Nature Mater. 8, 693 (2009).

[2] G. Witte, S. Lukas, P. S. Bagus, and C. Wöll, Appl. Phys. Lett. 87, 263502 (2005).
[3] L. Romaner, G. Heimel, J-L. Brédas, A. Gerlach, F. Schreiber, R. L. Johnson, J. Zegenhagen, S. Duhm, N. Koch, and E. Zojer, Phys. Rev. Lett. 99, 256801 (2007).

[4] T. Zhang, Z. P. Liu, S. M. Driver, S. J. Pratt, S. J. Jenkins, and D. A. King, Phys. Rev. Lett. 95, 266102 (2005).

[5] P. S. Bagus, D. Käfer, G. Witte, and C. Wöll, Phys. Rev. Lett. 100, 126101 (2008).

[6] P. S. Bagus, C. Wöll, and A. Wiechowski, Surf. Sci. 603, 273 (2009).

[7] G. Alexandrowicz, A. P. Jardine, H. Hedgeland, W. Allison, and J. Ellis, Phys. Rev. Lett. 97, 156103 (2006).

[8] G. Alexandrowicz, P. Kole, E. Lee, H. Hedgeland, R. Ferrando, A. P. Jardine, W. Allison, and J. Ellis, J. Am. Chem. Soc. 130, 6789 (2008).

[9] D. H. Sun, B. E. Bent, and J. G. Chen, J. Vac. Sci. Technol. A 15, 1581 (1997).

[10] D. Farías and K.-H. Rieder, Rep. Prog. Phys. 61, 1575 (1998).

[11] F. Mezei Neutron Spin Echo, Lecture Notes in Physics (Springer, New York, 1980).

[12] A. P. Jardine, H. Hedgeland, G. Alexandrowicz, W. Allison, and J. Ellis, Prog. Surf. Sci. 84, 323 (2009); A. P. Jardine, J. Ellis, and W. Allison, J. Chem. Phys. 120, 8724 (2004); A. P. Jardine, G. Alexandrowicz, H. Hedgeland, R. D. Diehl, W. Allison, and J. Ellis, J. Phys. Condens. Matter 19, 305010 (2007).

[13] L. Van Hove, Phys. Rev. 95, 249 (1954).

[14] G. L. Squires, Introduction to the Theory of Thermal Neutron Scattering (Cambridge University Press, Cambridge, England, 1978).

[15] S. Miret-Artes and E. Pollak, J. Phys. Condens. Matter 17, S4133 (2005).

[16] F.E. Tuddenham, H. Hedgeland, A. P. Jardine, B. A. J. Lechner, B. J. Hinch, and W. Allison, Surf. Sci. 604, 1459 (2010).

[17] J. W. Rowe, K. Sköld, H.E. Flowtow, and J. J. Rush, J. Phys. Chem. Solids 32, 41 (1971).

[18] H. Hedgeland, P. R. Kole, H. R. Davies, A. P. Jardine, G. Alexandrowicz, W. Allison, J. Ellis, G. Fratesi, and G. P. Brivio, Phys. Rev. B 80, 125426 (2009).

[19] S. J. Clark, M. D. Segall, C. J. Pickard, P. J. Hasnip, M. J. Probert, K. Refson, and M. C. Payne, Z. Kristallogr. 220, 567 (2005).

[20] J. P. Perdew, J. A. Chevary, S. H. Vosko, K. A. Jackson, M. R. Pederson, D. J. Singh, and C. Fiolhais, Phys. Rev. B 46, 6671 (1992).

[21] For $(2 \sqrt{3} \times 2 \sqrt{3})$ cell $(a=8.867068, b=8.867068, c=$ $\left.54.289210 \AA ; \alpha=90^{\circ}, \beta=90^{\circ}, \gamma=90^{\circ}\right)$, Cp neutral cubic cell length $10 \AA$. Convergence criteria: total energy convergence tolerance $=0.2000 \times 10^{-4} \mathrm{eV} /$ atom, maximum ionic force tolerence $=0.5000 \times 10^{-1} \mathrm{eV} / \AA$, maximum ionic displacement tolerence $=0.1000 \times$ $10^{-2} \AA$.

[22] R. Kubo, Rep. Prog. Phys. 29, 255 (1966).

[23] W. Kohn and K.H. Lau, Solid State Commun. 18, 553 (1976).

[24] J. Topping, Proc. R. Soc. A 114, 67 (1927).

[25] H. Hedgeland, P. Fouquet, A.P. Jardine, G. Alexandrowicz, W. Allison, and J. Ellis, Nature Phys. 5, 561 (2009).

[26] P. Hänggi, P. Talkner, and M. Borkovec, Rev. Mod. Phys. 62, 251 (1990). 